\title{
Entangled Nanoparticles: Discovery by Visualization in 4D Electron Microscopy
}

\author{
Aycan Yurtsever, J. Spencer Baskin, and Ahmed H. Zewail*
}

Physical Biology Center for Ultrafast Science and Technology, Arthur Amos Noyes Laboratory of Chemical Physics, California Institute of Technology, Pasadena, California 91125, United States

\section{Supporting Information}

ABSTRACT: Particle interactions are fundamental to our understanding of nanomaterials and biological assemblies. Here, we report on the visualization of entangled particles, separated by as large as $70 \mathrm{~nm}$, and the discovery of channels in their near-fields. For silver nanoparticles, the induced field of each particle extends to $50-100 \mathrm{~nm}$, but when particles are brought close in separation we observe channels as narrow as 6 $\mathrm{nm}$, a width that is 2 orders of magnitude smaller than the incident field wavelength. The channels' directions can be controlled by the polarization of the incident field, particle size, and separation. For this direct visualization of these nano-

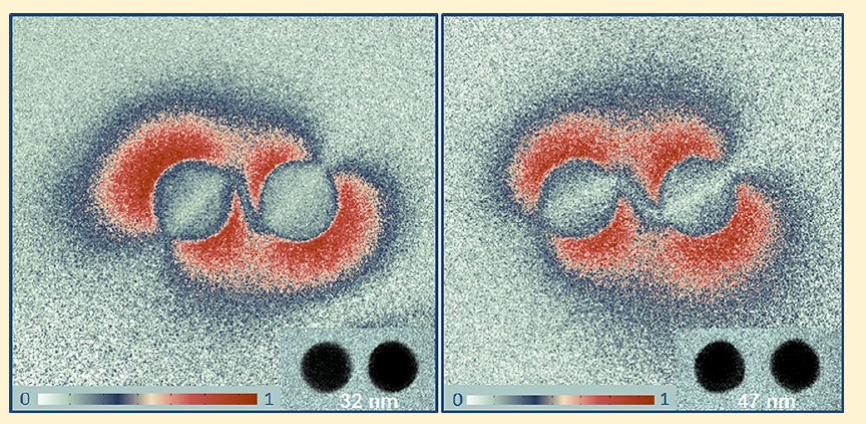
scopic near-fields, the high spatial, temporal, and energy resolutions needed were hitherto not possible without the methodology given here. This methodology, we anticipate, paves the way for further fundamental studies of particle entanglement and for possible applications spanning materials and macromolecular assemblies.

KEYWORDS: plasmonics, near-fields, ultrafast, electron microscopy, PINEM

$\mathrm{N}$ anoparticles at nanoscale separations can, in principle, interact with each other, and if this interaction is coherent, constructive or destructive interferences may result in an entanglement through their fields. Visualization of such entanglement of electric fields on the nanometer scale is a nontrivial task, mainly because the wavelength of the optical field used is hundreds of nanometers whereas the scale for the interference is only a few nanometers. Moreover, it is essential that the resolution in both space and time be appropriate for visualization to be achieved. If realized, however, the imaged electric potentials with a high field gradient can be manipulated for different particle distribution, shape, and orientation with possible implications for various applications such as, for example, trapping with ponderomotive force, ${ }^{1}$ optical tweezers, ${ }^{2}$ design of photonic and plasmonic devices, ${ }^{3}$ and even biological communications. ${ }^{4}$ It is, therefore, fundamental to observe these fields when particles approach each other and to uncover the nature of interactions between them.

Here, we report the visualization of particle-particle interaction and the discovery of particle entanglement with void-field channels in the observed electric field between them. The induced near-fields of nanoparticle pairs and chains were imaged using 4D electron microscopy, ${ }^{5}$ thus enabling the nanometer precision and femtosecond time resolution for visualization. Since the response of the nanoparticle system is to the linear polarization of the incident field, it was possible to control the dipolar particle polarization. For nanoparticles of typically $50 \mathrm{~nm}$ radius, the entanglement, despite the diffuseness of the field, results in very narrow channels, as narrow as $6 \mathrm{~nm}$ in width, an order of magnitude smaller than the spatial extent of the field for individual particles and 2 orders of magnitude smaller than the wavelength of the incident field. The channel orientation and shape can be tuned with the linear polarization of the exciting field, the size of the particle, and separation between them.

The above-mentioned visualizations are not possible without the technique of photon-induced-near-field-electron-microscopy (PINEM). ${ }^{6-8}$ When convergent nanoscale electron beams are used, instead of parallel beams, it is possible to form ultrafast spectrum images to map out the near-fields with subparticle resolution. ${ }^{9,10}$ Both methods provide the very high spatial $(\mathrm{nm})$ resolution but with the energy resolution and selectivity of optical techniques $(\mathrm{meV})$ and with the temporal resolution being in the femtosecond ( $f s$ ) domain. By comparison, light-based methods, such as near-field scanning optical microscopy, ${ }^{11,12}$ have spatial resolutions in the hundred nanometer range. ${ }^{13}$ On the other hand, conventional electron microscopic techniques, such as energy-filtered (parallel beam) imaging $^{14,15}$ and spectrum (convergent beam) imaging, ${ }^{16-18}$ have the needed spatial resolution, down to the atomic scale ${ }^{17}$ but without the capabilities of polarization, ultrafast temporal, and selective-excitation control.

Received: July 30, 2012

Published: August 8, 2012 


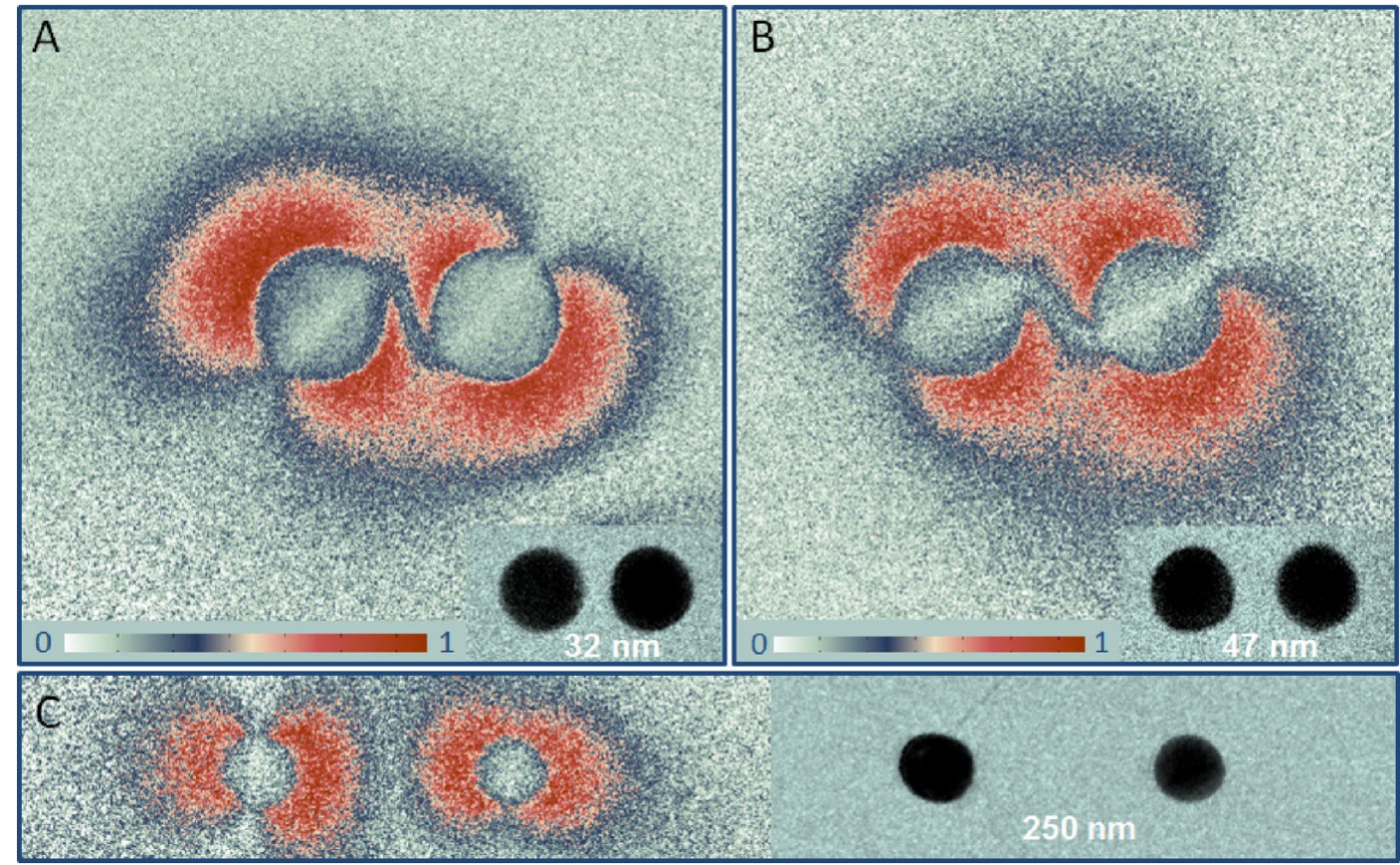

Figure 1. Entangled particles by dipolar fields and nanometer-scale void-channels. Shown are the near-fields of a nanoparticle pair with an edge-toedge distance of $32 \mathrm{~nm}$ (A), $47 \mathrm{~nm}$ (B), and $250 \mathrm{~nm}$ (C) with false-color mapping. The well separated particles in (C) exhibit dipolar-like fields and at this $250 \mathrm{~nm}$ separation they do not interact. In contrast, when the separation is reduced to $32 \mathrm{~nm}$ or $47 \mathrm{~nm}$ a "channel" is formed between the particles as seen in panels A and B. The bright field images of the nanoparticle pairs (obtained in UEM) are shown in the inset at the bottom right of panels A and B; PINEM images are displayed at a higher magnification than the bright-field UEM images in order to emphasize the particles entanglement. The magnification in (C) is half of that in the main panels A and B, and it is the same for both the PINEM and UEM images. The false-color bars are shown at the bottom left of panels A and B; white indicates the lowest intensity and red the highest. Polarization of the exciting laser field is at $45^{\circ}$ counterclockwise (see Figure 2) for panels A and B, and it is horizontal for (C).

Here, an ultrashort (fs) optical pulse excites the material and induces near-fields in the vicinity of nanoparticles. Synchronously, an ultrashort (fs) electron pulse images the induced field that rises and falls on the fs time scale. It is this femtosecond time scale that enables the arresting of the field at high peak amplitudes. An inelastic energy exchange between the temporally arrested field and the ultrafast electron results in electron energy-gain (absorption) or energy-loss (emission) at integer multiples of the photon energy. Since these inelastic scatterings take place only in the presence of the near-field, it is possible to map out the spatial distributions of these fields by recording the scattered electrons. This is achieved by a postcolumn spectrometer in our microscope, where only the energy-gained electrons are selected to form the image; see Supporting Information for the experimental methods. We note that in PINEM, the excitation and polarization of the field are controllable features, while in conventional electron microscopy methods, the fields are induced by the imaging electrons. For this electron excitation case, all modes of the near-field may be produced, and entanglement may be obscured.

When two nanoparticles are brought in close proximity to each other, as shown in Figure 1, entanglement may take place through their fields. The total electric field at any point in space $(x, y, z)$ and at time $(t)$ is the coherent vectorial sum of the particles' fields, that is

$$
\vec{E}_{\text {total }}^{\text {coherent }}=\vec{E}_{1}(x, y, z ; t)+\vec{E}_{2}(x, y, z ; t)
$$

It follows that interference of the particles' fields described vectorially in eq 1 will result only when phases are preserved, the coherent regime $\left|E_{1}+E_{2}\right|$, as oppose to the case of the incoherent addition of fields, $\left|E_{1}\right|+\left|E_{2}\right|$. At separations larger than the field decay length of a single particle, the electric fields do not interact significantly, and the observed images are those of two separate dipoles. This is shown in Figure 1 C, in which the particles are separated by $250 \mathrm{~nm}$. It is evident that the two dipolar lobes of each particle are similar in size, which demonstrates that the near-field interaction is nearly absent.

On the other hand, when particle separation becomes comparable to or less than the decay length, the near-fields interfere and channels open up between the particles, as shown in Figure 1A,B. The shape and width of this unique channel depend on the particle separation and polarization. As the edgeto-edge distance increases from 32 to $47 \mathrm{~nm}$, the width of the channel was observed to increase from 11 to $20 \mathrm{~nm}$; the channel eventually disappears at large separations. Importantly, these channels are formed in the space between the two particles. As seen in the bright-field images (insets in Figure 1 $A, B)$, there is nothing in the space between the particles, yet, in the PINEM images high contrast is observed in the in-between space.

In Figure 2, we display the polarization effect and the change of the channel orientation for various angles. The change in contrast for all polarizations can be understood by considering the interference (or superposition) of the electric fields from the two particles, eq 1 . Contrast is formed as a result of an energy exchange between the ultrafast electrons and the nearfield component along the electron trajectory $\left(E_{z}\right)$. At the center point between the dipoles (Figures 1 and 2), the $E_{z}$ contributions from the two particles are the same in amplitude and of opposite sign (i.e., they are out of phase) and, hence, they cancel each other. For the rest of the points on the axis of the nanoparticle pair (that is along the line connecting the two 

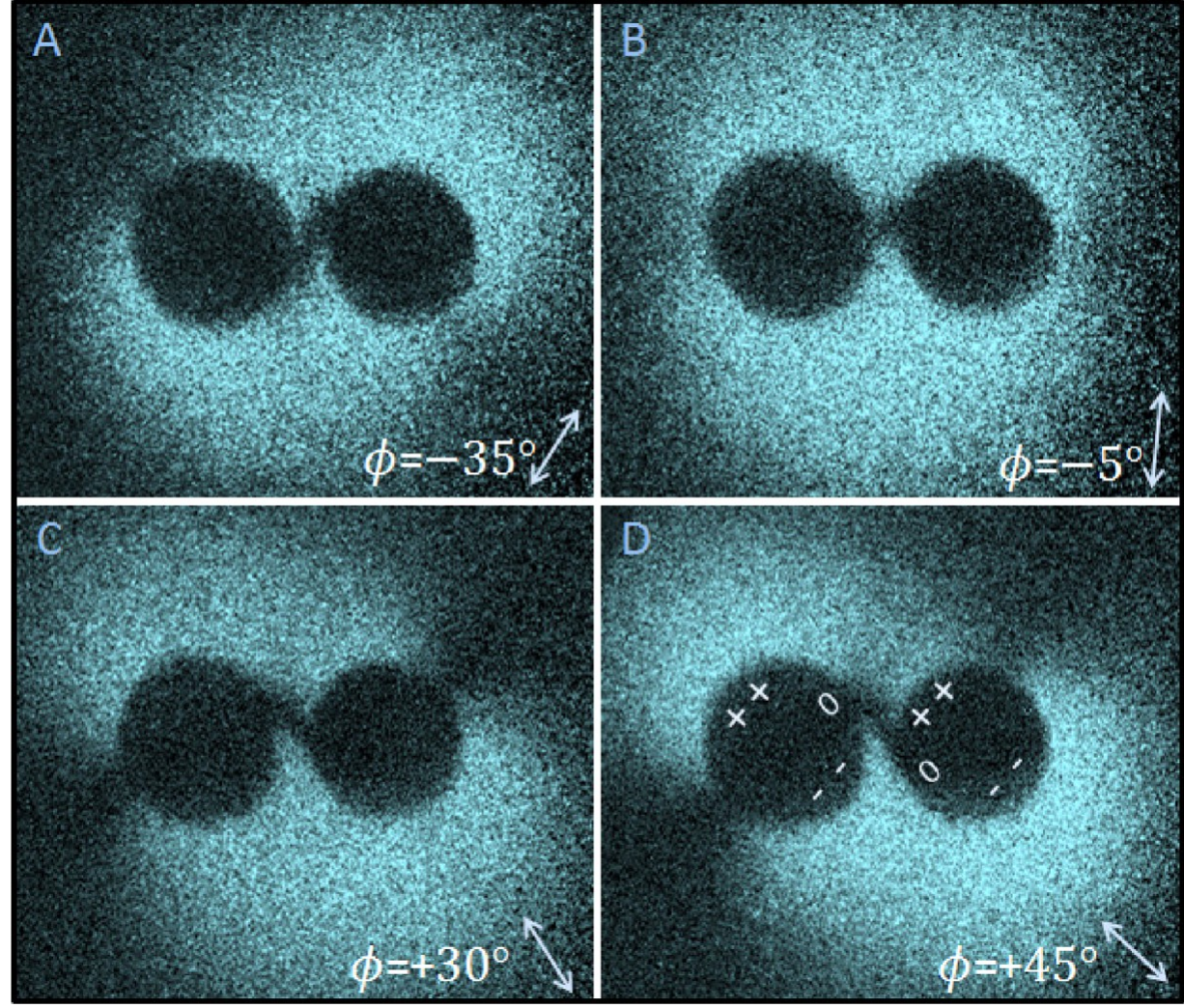

Figure 2. Polarization dependence of the entanglement. When the exciting optical pulse is polarized vertically, the channel is only along the horizontal direction and the resulting structure looks like a "dumbbell" (B). When the linear polarization is rotated on either side, the channels observed in Figure 1 are retrieved, $(A, C)$. Induced electric charge distributions on the nanoparticles are indicated in (D), and it is seen that the voidchannel is "attached" to the particles near the zero-charge points. Particle separation is $20 \mathrm{~nm}$ (edge-to-edge). Intensities are shown in a gray scale with a blue hue for clearer visualization. The intensity values for each image are normalized/stretched to fit the same intensity scale (0-255) after correcting for the top and bottom $1 \%$ intensity-outliers.

particles), the $E_{z}$ contributions are unequal (except for the case of Figure 2B; see below) and, hence, the total $E_{z}$ is nonzero. Therefore, for the induced polarizations depicted in Figure $1 \mathrm{~A}, \mathrm{~B}$ and Figure 2A,C,D, the channel is not a straight connection between the two particle edges but rather turns and follows the orientation of the linear polarization, as discussed below. We note that the contrast inside the nanoparticle is determined mostly by the diffraction loss of the transmitted electrons through the nanostructure; see Supporting Information.

Further insight into the mechanism of channel formation can be gained by analyzing the channel polarization dependencies. In Figure 2, the linear polarization of the excitation pulse was rotated in the lateral plane, and the figure shows the entanglement at four different polarization angles (particle separation is $20 \mathrm{~nm}$ ). When the electric field of the $519 \mathrm{~nm}$ laser is aligned vertically $\left(\varphi=0^{\circ}\right)$, the channel stretches between the nearest points of the particles, and the resulting shape resembles a "dumbbell", very similar to the experimental observation close to $\varphi=0^{\circ}\left(\varphi=-5^{\circ}\right)$. When it is rotated to $\varphi$ $=-35^{\circ}$ (clockwise) and $\varphi=+30^{\circ}$ (counter-clockwise), shapes similar to those presented in Figure 1 are retrieved, with the twist of the void-channel following the direction of the polarization vector.

This observed polarization behavior can be understood by considering the induced electric charge distributions, which are shown schematically at the peak of one optical cycle in Figure 2D for the $\varphi=+45^{\circ}$ polarization. This distribution is dipolar in nature because the particles are small compared to the wavelength of the exciting field: this field drives the conduction band electrons of the silver particle to create plus (+) and minus ( -$)$ charge accumulations at the opposite surfaces of the particle, while the plane that is orthogonal to the polarization vector (and passes through the center of particle) remains without any net charge. We call points on this latter plane at the boundary of the particle the zero-charge-points, one of which for $z=0$ is shown for each particle in Figure 2D. Both particles in the pair have the same symmetry in their charge distributions; here the effect of the induced field of one particle on the charges of the other particle is not considered.

As we rotate the polarization, the zero-charge-points move on the perimeter of the disk defined by the lateral cross section of the particle. Importantly, the motion of the points on the adjacent edges of the two particles will be in opposite directions. In the dipole approximation, which is satisfied here, the $E_{z}$ of individual particle fields are null on their zerocharge-points. Therefore the total $E_{z}$ field approaches zero at these points. Since $E_{z}$ also equals zero at the high-symmetry point mentioned above (the center point between the two particles), it follows from the continuity of the fields that a void-channel connecting these three points will be formed. Indeed, this is what is experimentally observed: the joining points of the channel to the particles are near the zero-charge points. As for the polarization dependence, since the two points (the zero-charge points) move with the polarization direction 

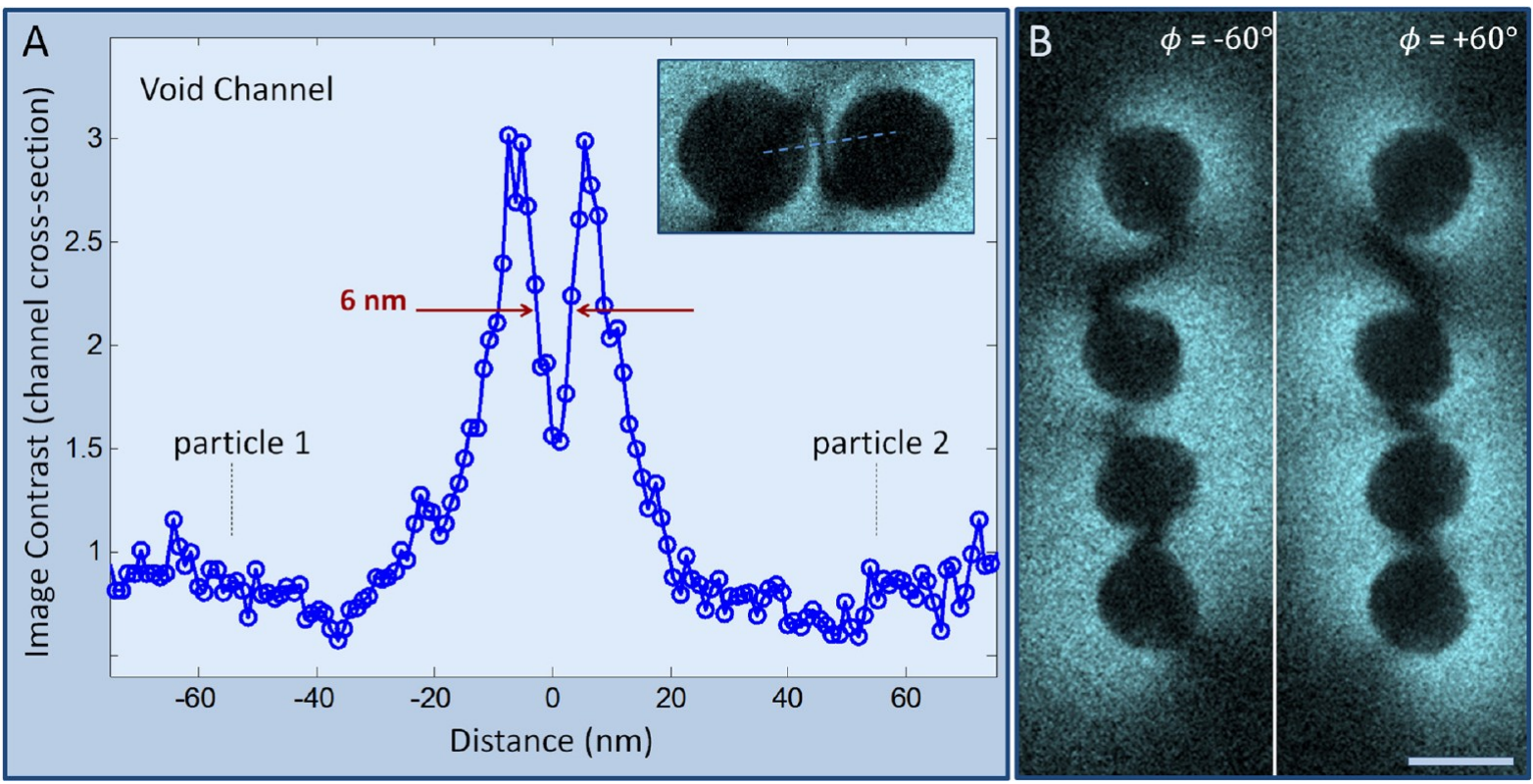

Figure 3. Spatial extent of entanglement and channels of particle chains. The cross section across the channel of two entangled particles (separated by $14 \mathrm{~nm}$, edge-to-edge) is shown in (A), where the image intensity is plotted as a function of distance along the dashed line shown in the inset; the centers of the two particles involved are given as dashed black lines. It is evident that the width of the channel is only $6 \mathrm{~nm}$, implying large electric field gradients (see text). The near-fields and their interactions for a chain of four particles are shown in (B); the scale bar in (B) is $100 \mathrm{~nm}$.

while the third point (center of the two particles) remains the same, the channel rotates with the rotating polarization.

The striking very narrow width of the channel, as a result of the entanglement, is displayed in Figure 3A. Shown together with the PINEM image is a cross-section of the channel taken at its center and in the perpendicular direction (see the dashed line in the inset); the nanoparticle separation in this case is 14 $\mathrm{nm}$. The cross-sectional plot depicts the width of the channel, 6 $\mathrm{nm}$, which is only $1 \%$ of the excitation wavelength $(519 \mathrm{~nm})$ that creates the near-fields. At our incident fs-pulse peak fluence $\left(3 \mathrm{~mJ} / \mathrm{cm}^{2}\right)$ at the specimen, the field is $3 \times 10^{6} \mathrm{~V} / \mathrm{cm}$, giving a maximum gradient of the calculated induced field of $10^{5} \mathrm{~V} / \mathrm{cm}$ per nm for the channel.

For a chain of particles, Figure $3 \mathrm{~B}$ shows the near-fields observed at two different polarizations. The presence of entanglement is apparent in the gaps between the particles. Again, the polarization dependence is consistent with the results of the two-particle entanglement discussed above. From the fields between the top two particles it is evident that the channels are observable even when the particle separation is 70 $\mathrm{nm}$. As importantly, for an assembly of particles one can manipulate their directions and presence by controlling the polarization and/or particle separation.

For quantification of the observed experimental images, we calculated the electric fields expected as well as the PINEM fields. The results are given in Figure 4. Because the dipole mode is dominant in the image, the particle can be replaced in the calculations with a point dipole placed at its center. Other modes and fields are inherently excluded in the dipole (Rayleigh) case; for the more general treatment Mie theory can be invoked. The field component relevant here $\left(E_{z}\right)$ and its spatial extent in the image $(x-y)$ plane at various height $(z)$ values can be written as

$$
E_{z}(x, y, z ; t) \approx E_{0} a^{3} \chi(\omega) \frac{3 x z}{r^{5}} e^{-i \omega t}
$$

for $x$-polarization and with $r=\left(x^{2}+y^{2}+z^{2}\right)^{1 / 2}$. In eq $2, E_{0}$ is the amplitude of the incident field, $a$ the particle radius, and $\chi(\omega)=[\varepsilon(\omega)-1] /[\varepsilon(\omega)+2]$ where $\varepsilon(\omega)$ is the complex dielectric function of the material (silver in this case) at the incident field's frequency $(\omega)$. Note that since $\varepsilon(\omega)$ is in general a complex quantity, $E_{z}$ (even at $t=0$ ) will be complex as well, having a well-defined amplitude and phase.

Figure 4A gives the calculated $E_{z}$ value in the $x-y$ plane for three different $z$ values, and for a single and pairs of interacting particles. Fields are calculated for each particle using eq 2 and the entangled fields of two particles are obtained using eq 1 . Here, $t=0$ and the polarization is rotated from the vertical axis $\left(\varphi=+45^{\circ}\right)$. The last term in eq 2 represents the temporal cycling of the field; the experimental delay time is incorporated in $E_{0}$ and defines the $t=0$ for imaging in PINEM. Single particle images show the dipolar lobes and their strength is minimum when $z$ is close to zero $(z=0$ is the plane that passes through the center of the particles and is orthogonal to the trajectory of incident photons and electrons along $z$ ). As the $z$ value increases, the lobe intensity increases $(z=20 \mathrm{~nm})$ but then falls for larger values $(z=50 \mathrm{~nm})$; the same behavior is reproduced in the lower half-space, that is, for $z<0$, due to the symmetry in $z$, eq 2 . Interaction of the two particles is calculated from the single particle dipole fields but using the superposition of the fields. The results are presented in the bottom row of Figure 4A. Indeed, the channels emerge in the near-field region between the particles.

In order to compare the fields to the observed images we evaluated the PINEM field, which can simply be obtained by integrating $E_{z}\left(x, y, z ; t=t_{\mathrm{e}}\right)$ along $z$, from $-\infty$ to $+\infty$. $^{7}$ This is equivalent to the work done on the electron by the induced electric field. Because $E_{z}$ oscillates in time (the $e^{-i \omega t}$ term) the field couples to the electron's motion during its transit time, that is, when $t_{\mathrm{e}}=(z / v)$, where $v$ is the speed of the electron, in this case $0.7 c$ at $200 \mathrm{keV}$. This substitution enables calculation of the PINEM field, and in this case it is simply 
A. Electric Fields

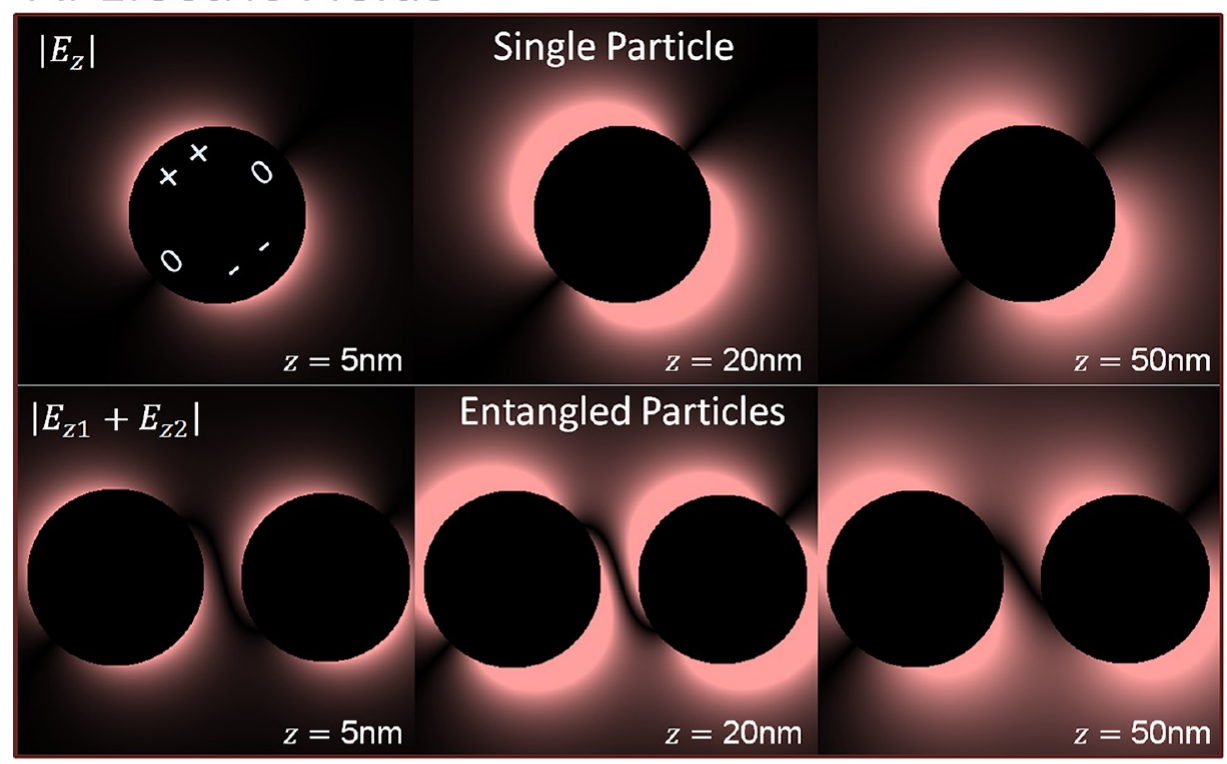

\section{B. PINEM Fields}

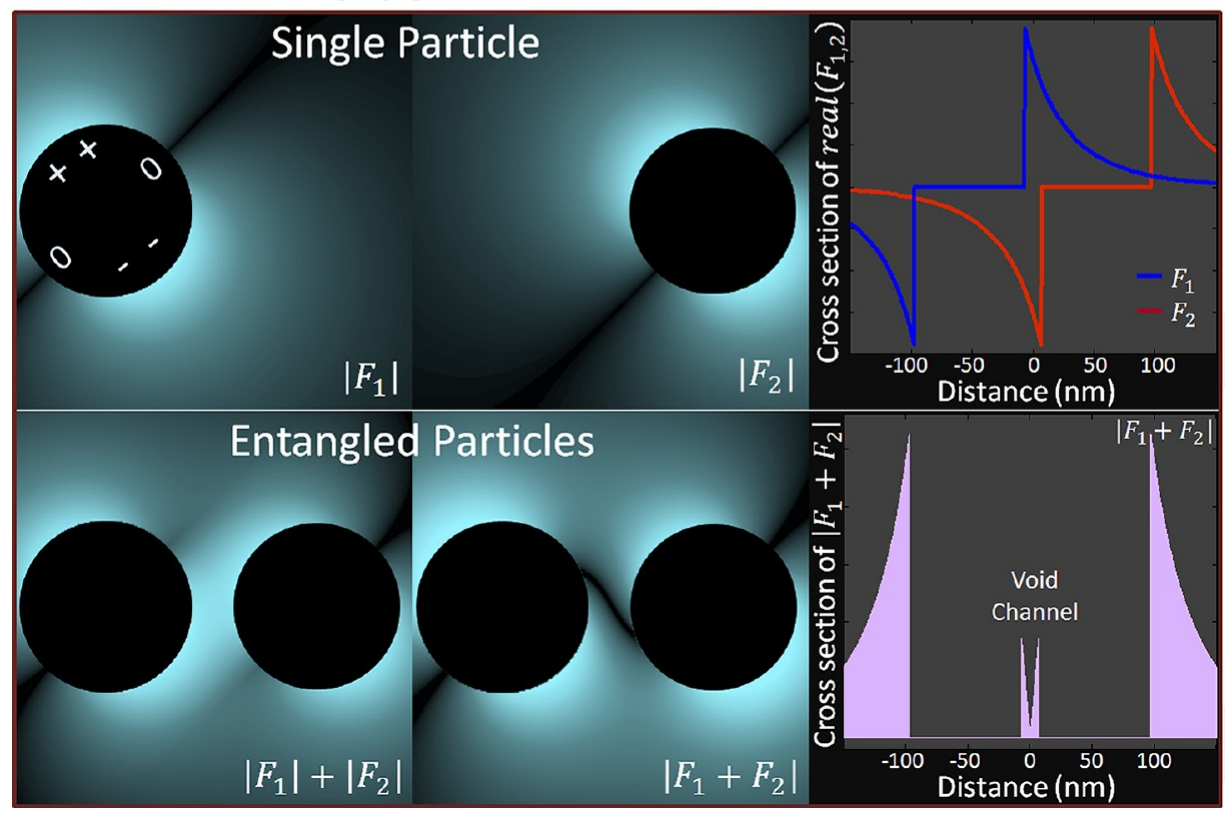

Figure 4. Theoretical calculations of fields of single and interacting particles. The exciting photon propagation is along $z$ (perpendicular to the page) and its polarization is at $45^{\circ}$ along the $(++)$ and (--) charges shown. Near field spatial distribution of $\left|E_{z}\right|$ of a nanoparticle in the lateral $x-y$ plane is shown at the top row of (A) for different height $(\boldsymbol{z})$ values. The bottom row presents the fields of two interacting nanoparticles, where the formation of void-channels is clearly visible. The PINEM fields and their cross-section along the interparticle axis are shown in (B) for single and interacting particles. These fields are connected to the electric fields shown in (A) through a Fourier transformation (see text). Importantly, when the individual PINEM fields of two particles are summed incoherently (bottom-left) the void-channels are absent. They are formed only when the superposition is coherent (bottom-middle). The cross sections of $F(x, y)$ of the individual particles (top-right) indicate that the real parts of the fields are out-of-phase between the particles, and the coherent superposition of these fields results in the void-channel (bottom-right). Image intensities are given in a gray scale but they are presented in red (A) and nile-blue (B) colors for visualization. Particle positions are shown with zero-intensity (black) discs in all images.

$$
F(x, y) \approx \int_{-\infty}^{+\infty} E_{z}(x, y, z) e^{-i(\omega / v) z} \mathrm{~d} z
$$

which states that PINEM fields are the Fourier transform of $E_{z}(x, y, z ; t=0)$ at a fixed component of $\omega / v$, a consequence of momentum conservation in the scattering process. ${ }^{7,8}$ The expression for the PINEM intensity involves the square of $F(x$, y). ${ }^{19}$
Figure 4B (top row) depicts the field $F(x, y)$ for two single particles together with the cross sections along the horizontal axis through the centers of the particles. The images depict the magnitude of $F(x, y)$, and they closely follow the $x-y$ distribution of the electric fields (Figure 4A). Shown in the bottom row of Figure $4 \mathrm{~B}$ is the case of two interacting particles. The channel is evident only when the coherent superposition is appropriately calculated with the phases included $\left|F_{1}+F_{2}\right|$, not 
the incoherent sum $\left|F_{1}\right|+\left|F_{2}\right|$. To elucidate the origin of the channel narrowness it is helpful to plot the cross-sectional real parts. The panel on the right (Figure 4B) indicates the opposite sign for each particle's field (top), and the very narrow spatial change of the field sign (between particles) to yield the narrow channel, as observed experimentally. When the incoherent summation is performed, $\left|F_{1}\right|+\left|F_{2}\right|$, the channels are no longer present and the particles do not entangle.

The observed void-channels are in the $z$-component of the field, and future experiments, using the tomography-like approach and/or varying the electron velocity, can similarly enable the visualization of the other components, giving a related anisotropic picture of the total field, in analogy with orbitals contributions in atomic bonding. Depending on particles shapes and field distributions, the spatial characteristics of the total field might be tailored by arranging several nanoparticles on a two-dimensional plane, or even in a threedimensional space. Similarly, more than one laser excitation, with the freedom to tune their polarizations, wavelengths, and directions, could be employed to, for instance, trap and guide particles/molecules along desired directions. Here the nearfield, when the particles are interacting, is enhanced by an order of magnitude reaching peak values of $\sim 10^{11} \mathrm{~W} / \mathrm{cm}^{2}$, suggesting applications for devices such as nano-antennas. ${ }^{20}$

The discovery of nanoscale channels in the fields of entangled particles, together with the ability to visualize them in space and time, provide the opportunity to unravel fundamentals of microscopic interactions between particles of materials and of biological assemblies. These imaging methods should find applications in nanoscale materials science ${ }^{21-24}$ and possibly in the study of biological channels and selfassembly. ${ }^{25,26}$

\section{ASSOCIATED CONTENT}

\section{S Supporting Information}

Additional information. This material is available free of charge via the Internet at http://pubs.acs.org.

\section{AUTHOR INFORMATION}

\section{Corresponding Author}

*E-mail: zewail@caltech.edu.

\section{Notes}

The authors declare no competing financial interest.

\section{ACKNOWLEDGMENTS}

This work was supported by the National Science Foundation (DMR-0964886) and the Air Force Office of Scientific Research (FA9550-11-1-0055) in the Gordon and Betty Moore Center for Physical Biology at the California Institute of Technology. We thank Dr. Sang Tae Park for helpful discussions and Dr. Renske van der Veen for the initial collaboration that resulted in the publication of ref 9 .

\section{REFERENCES}

(1) Ashkin, A. Trapping of Atoms by Resonance Radiation Pressure. Phys. Rev. Lett. 1978, 40, 729-732.

(2) Grier, D. G. A revolution in optical manipulation. Nat. Photonics 2003, 424, 810-816.

(3) Prasad, P. N. Nanophotonics; Wiley-Interscience: Hoboken, NJ, 2004.

(4) von Maltzahn, G.; Park, J.-H.; Lin, K. Y.; Singh, N.; Schwöppe, C.; Mesters, R.; Berdel, W. E.; Ruoslahti, E.; Sailor, M. J.; Bhatia, S. N.
Nanoparticles that communicate in vivo to amplify tumour targeting. Nat. Mater. 2011, 10, 545-552.

(5) Zewail, A. H. Four-Dimensional Electron Microscopy. Science 2010, 328, 187-193.

(6) Barwick, B.; Flannigan, D. J.; Zewail, A. H. Photon-induced nearfield electron microscopy. Nature 2009, 462, 902-906.

(7) Park, S. T.; Lin, M. M.; Zewail, A. H. Photon-induced near-field electron microscopy (PINEM): theoretical and experimental. New J. Phys. 2010, 12, 123028.

(8) Yurtsever, A.; Zewail, A. H. Direct Visualization of Near-Fields in Nanoplasmonics and Nanophotonics. Nano Lett. 2012, 12 (6), 33343338.

(9) Yurtsever, A.; van der Veen, R. M.; Zewail, A. H. Subparticle Ultrafast Spectrum Imaging in 4D Electron Microscopy. Science 2012, $335,59-64$.

(10) Batson, P. E. Plasmonic Modes Revealed. Science 2012, 335, 47-48.

(11) Betzig, E.; Trautman, J. K. Near-Field Optics: Microscopy, Spectroscopy, and Surface Modification Beyond the Diffraction Limit. Science 1992, 257, 189-195.

(12) Paesler, M. A.; Moyer, P. J. Near-Field Optics; Wiley: New York, 1996.

(13) Rang, M.; Jones, A. C.; Zhou, F.; Li, Z.-Y.; Wiley, B. J.; Xia, Y.; Raschke, M. B. Optical Near-Field Mapping of Plasmonic Nanoprisms. Nano Lett. 2008, 8, 3357-3363.

(14) Batson, P. E. Spatial resolution in electron energy loss spectroscopy. Ultramicroscopy 1992, 47, 133-144.

(15) Ugarte, D.; Colliex, C.; Trebbia, P. Surface- and interfaceplasmon modes on small semiconducting spheres. Phys. Rev. B 1992, $45,4332-4343$.

(16) Nelayah, J.; Kociak, M.; Stephan, O.; Garcia de Abajo, F. J.; Tence, M.; Henrard, L.; Taverna, D.; Pastoriza-Santos, I.; Liz-Marzan, L. M.; Colliex, C. Mapping surface plasmons on a single metallic nanoparticle. Nat. Phys. 2007, 3, 348-353.

(17) Muller, D. A.; Kourkoutis, L. F.; Murfitt, M.; Song, J. H.; Hwang, H. Y.; Silcox, J.; Dellby, N.; Krivanek, O. L. Atomic-Scale Chemical Imaging of Composition and Bonding by AberrationCorrected Microscopy. Science 2008, 319, 1073-1076.

(18) García de Abajo, F. J. Optical excitations in electron microscopy. Rev. Mod. Phys. 2010, 82, 209-275.

(19) We note that the experimental PINEM intensities for different gain peaks are, in general, computed by using the square of the sum of the PINEM fields, eq 3, as an argument in Bessel functions. ${ }^{7}$ This procedure produces images that are similar to those shown in Figure 4B. For the case of very strong incident fields, saturation in PINEM intensities may occur, but such saturation makes the channels even narrower in width.

(20) Cubukcu, E.; Nanfang, Y.; Smythe, E. J.; Diehl, L.; Crozier, K. B.; Capasso, F. Plasmonic Laser Antennas and Related Devices. Selected Topics in Quantum Electronics, Journal of IEEE 2008, 14, 14481461.

(21) Cui, Y.; Lieber, C. M. Functional Nanoscale Electronic Devices Assembled Using Silicon Nanowire Building Blocks. Science 2001, 291, 851-853.

(22) Lieber, C. M. Nanoscale Science and Technology: Building a Big Future from Small Things. MRS Bull. 2003, 28, 486-491.

(23) Alivisatos, A. P. Semiconductor Clusters, Nanocrystals, and Quantum Dots. Science 1996, 271, 933-937.

(24) Yuk, J. M.; Park, J.; Ercius, P.; Kim, K.; Hellebusch, D. J.; Crommie, M. F.; Lee, J. Y.; Zettl, A.; Alivisatos, A. P. High-Resolution EM of Colloidal Nanocrystal Growth Using Graphene Liquid Cells. Science 2012, 336, 61-64.

(25) Jena, B. P.; Hörber, J. K. H. Force microscopy: applications in biology and medicine; Wiley-Liss: Hoboken, NJ, 2006.

(26) Whitesides, G. M.; Grzybowski, B. Self-Assembly at All Scales. Science 2002, 295, 2418-2421. 